\title{
UM SISTEMA HÍBRIDO DE APOIO À DECISÃO FORMADO POR REDES DE PETRI, SIMULAÇÃO E SISTEMA ESPECIALISTA
}

\author{
Fabiano A. Hennemann* \\ fandrecdas.ufsc.br
}

Ricardo J. Rabelo*

rabelo@das.ufsc.br

\author{
José V. Canto dos Santos ${ }^{\dagger}$ \\ jvcanto@unisinos.br
}

José E. R. Cury*

curydas.ufsc.br

${ }^{*}$ Departamento de Automação e Sistemas - UFSC CP 476, CEP 88040-900 - Florianópolis - SC, Brasil

${ }^{\dagger}$ PIPCA - UNISINOS CP 275, CEP 93022-000 - São Leopoldo - RS, Brasil

\section{ABSTRACT}

This work presents a Decision Support System (DSS) with a hybrid approach, using Simulation and coloured Petri Nets as modeling techniques of manufacturing processes, associated with an Expert System (ES) to help in their use. The DSS provides a friendly interface for the user who, after selecting input parameters, obtains sets of data about the manufacturing process. To validate the proposed DSS, tests have been made using a real plant of footwear manufacturing. The objectives of the case study were to elaborate a group of proposals for improving the productive system performance, to evaluate the impacts created by change on the model parameters and to provide a better understanding about the productive plant. The results offer assistance on decision making and documentation processes. The DSS renders possible for managers, without any knowledge of modeling techniques, to manipulate sets of data and to interact with the system. The developed model can be used for any industrial plant, provided that the model input parameters are adequately adjusted.

KEYWORDS: Simulation, Decision Support System, Petri

ARTIGO CONVIDADO:

Versão completa e revisada de artigo apresentado no CBA-2004

Artigo submetido em 15/12/04

1a. Revisão em 04/02/05

2a. Revisão em 12/07/05

3a. Revisão em 04/04/06

Aceito sob recomendação do Ed. Assoc. Prof. Paulo Eigi Miyagi
Nets, Expert Systems, Coloured Petri Nets

\section{RESUMO}

O objetivo do presente trabalho é apresentar um sistema híbrido para apoio à decisão (SAD), baseado em simulação e redes de Petri coloridas (CPN) como técnicas de modelagem de processos, associado a um Sistema Especialista (SE) como apoio à sua utilização. O SAD fornece uma interface amigável para o usuário que, após a seleção de parâmetros de entrada, obtém dados sobre o processo produtivo. Para validar o SAD proposto, testes foram realizados utilizando uma planta produtiva real do setor calçadista. Os objetivos do estudo de caso foram elaborar um grupo de propostas para aperfeiçoamento do desempenho do sistema produtivo; avaliar os impactos gerados pela mudança nos parâmetros do modelo; e fornecer uma melhor compreensão da planta produtiva. O SAD mostrou permitir que gestores, mesmo sem conhecimento de técnicas de modelagem, interajam com o sistema e manipulem conjuntos de dados. O sistema ainda mostrou poder ser utilizado para qualquer planta industrial, desde que os parâmetros de entrada do modelo sejam adequadamente ajustados.

PALAVRAS-CHAVE: Simulação, Sistemas de Apoio à Decisão, Redes de Petri Coloridas, Sistema Especialista. 


\section{INTRODUÇÃO}

As empresas devem constantemente aperfeiçoar seus processos de fabricação e seus métodos de trabalho. Para isto é necessário o aprimoramento do processo produtivo, procurando a redução do lead time, custos de produção e melhoria da qualidade, entre outros. Entretanto, é sabida a dificuldade para se atingir estes objetivos. Uma das causas é a carência de bons sistemas computacionais que auxiliem os gestores na avaliação das empresas e na posterior tomada de decisão. A existência de uma ferramenta de apoio à decisão que interaja com um modelo do processo de fabricação pode beneficiar estas empresas no sentido de analisar o desempenho de seus processos, determinar cronogramas de execução com maior precisão, relacionar as operações e o planejamento dos recursos necessários na fabricação de cada tipo de produto e analisar impactos de alterações em seu processo.

Este trabalho apresenta um sistema híbrido para apoio à decisão que combina as potencialidades de Redes de Petri, simulação e Sistemas Especialistas, conforme (Hennemann, 2004), visando melhorar a qualidade do diagnóstico e das decisões a serem tomadas. Com auxílio de uma ferramenta com esse perfil pode-se analisar a formação de filas, identificar possíveis gargalos do processo produtivo e aproveitar de forma otimizada os recursos disponíveis. No desenvolvimento deste trabalho foi escolhido o segmento de produção de matrizes para calçados para avaliar e validar o sistema desenvolvido. No setor calçadista, os processos podem ser considerados como sendo do tipo flow shop (Askin e Standridge, 1993), com grande variabilidade e produção do tipo média, conforme descrito em (Groover, 2001). A escolha de técnicas de modelagem onde exista interação com os gestores responsáveis pelos processos produtivos, como visto em (Piesik and Weglarz, 1999), (Shahraray and Maeschke, 1990), (Malmborg and Berrings, 1992) e (Reilly and Lilegdon, 1999) é um ponto importante no contexto deste trabalho. Ainda na literatura sobre a área perrcebe-se que diversos autores, como (Mielke, 1999), (Pool and Stafford, 1998) e (Matsuyama and Atherton, 1990), propõem o uso de simulação para o tratamento de sistemas de manufatura. Já o uso de CPNs é proposto em um número menor de trabalhos, sendo alguns exemplos os de (Zhang et alli, 2002), (Zimmermann et alli, 1996), (Narciso, 2001), (Descotes, 1994) e (Jeng et alli, 1997).

O artigo está organizado da forma descrita a seguir. Após esta introdução, a seção 2 detalha o problema enfocado no trabalho. A seção 3 mostra o sistema proposto, cuja implementação está na seção 4 . Já a seção 5 contém os diversos testes realizados bem como a análise dos resultados obtidos. Encerrando, a seção 6 apresenta as conclusões do trabalho.

\section{PROBLEMA ENFOCADO}

Sistemas de apoio à decisão são sistemas computacionais interativos que têm como objetivo auxiliar tomadores de decisão a utilizar dados, modelos e estruturas de maneira que estes possam ajudar a resolver problemas e tomar decisões (Bohanec, 2001). O problema enfocado neste trabalho é a necessidade de construção de um sistema computacional híbrido para apoio à decisão em ambientes industriais. Sendo híbrido, espera-se que tal sistema combine as características de várias técnicas de modelagem e avaliação de desempenho. Alguns subproblemas constatados, formadores do problema enfocado são listados a seguir.

- A seqüência de fabricação de produtos não costuma estar documentada, dificultando a visualização, compreensão e análise do processo. (A necessidade de elaborar documentação vem do fato de que a maioria das empresas visa a implantação de processos de certificação.)

- Existem poucas estimativas dos tempos precisos de fabricação. Isto prejudica diretamente o cronograma e os cálculos dos custos de produção.

- Não existe estimativa confiável de utilização dos recursos disponíveis, se estão ociosos, sobrecarregados ou provocando gargalos no processo.

- Ausência de uma ferramenta para analisar impactos dos investimentos em novos recursos e quais as conseqüências de alterações na seqüência das etapas .

- Grande quantidade de retrabalho em conseqüência da despadronização da seqüência produtiva.

\section{SISTEMA PROPOSTO}

A Figura 1 mostra a arquitetura básica do sistema proposto, destacando-se nela o uso de simulação, CPNs e de um sistema especialista. Sabe-se que simulação é adequada para avaliar o processo produtivo de empresas através de cenários propostos. Sua utilização permite entender relações de causa / efeito das decisões, responder questões what-if, prover ambientes virtuais de testes e melhorar o desempenho da planta. Já CPNs são eficientes no planejamento da estrutura do processo e no planejamento da produção proporcionando eficientes análises estruturais. Sistemas especialistas sintetizam, em sua base de conhecimento a experiência de pessoas que conhecem bem os processos em estudo. A lógica de comunicação entre os módulos está baseada na leitura e escrita de arquivos texto e planilhas de dados. Para visualização da comunicação entre os módulos do SAD, a Figura 2 mostra o diagrama de seqüência do sistema. 


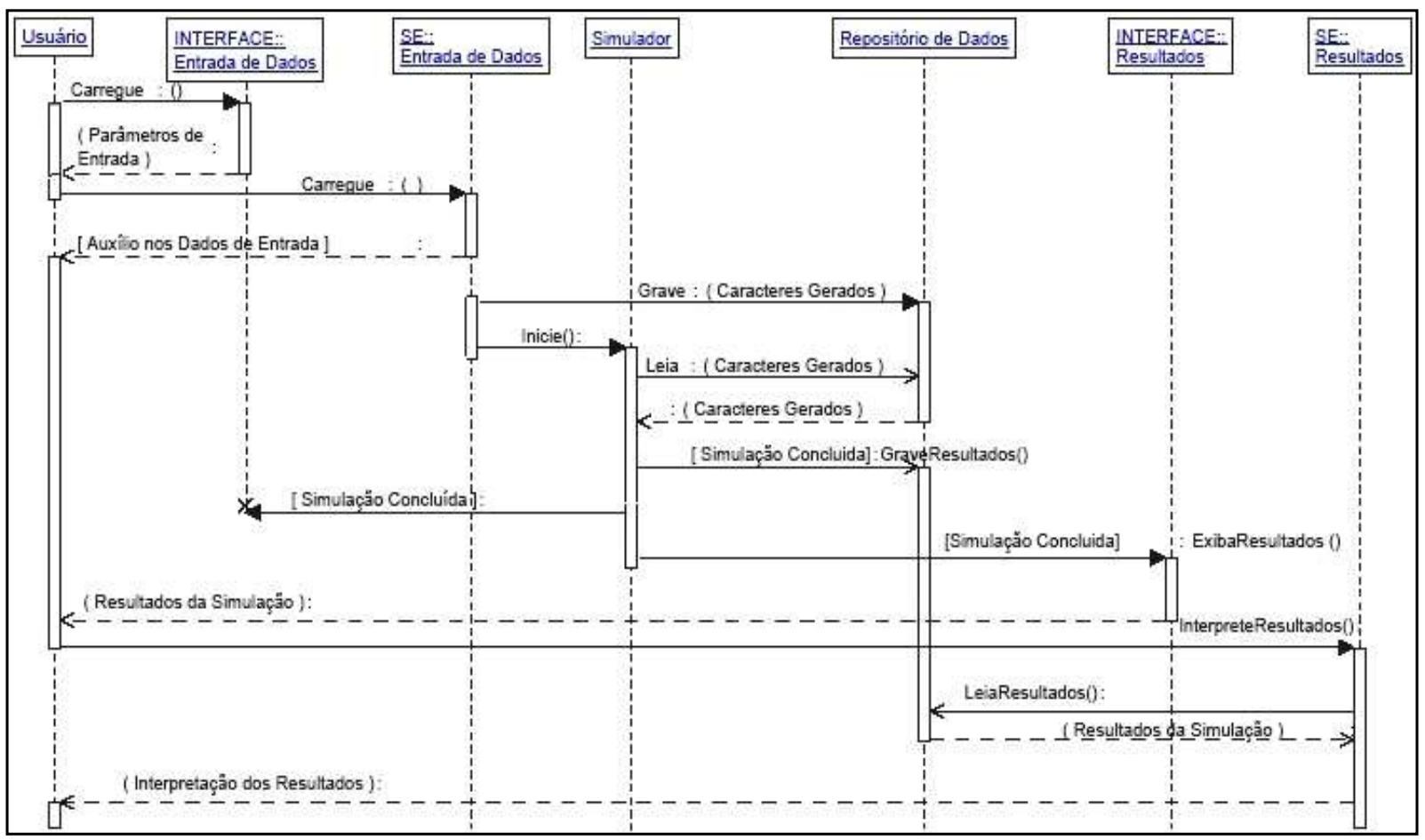

Figura 2: Diagrama de Seqüência dos Módulos

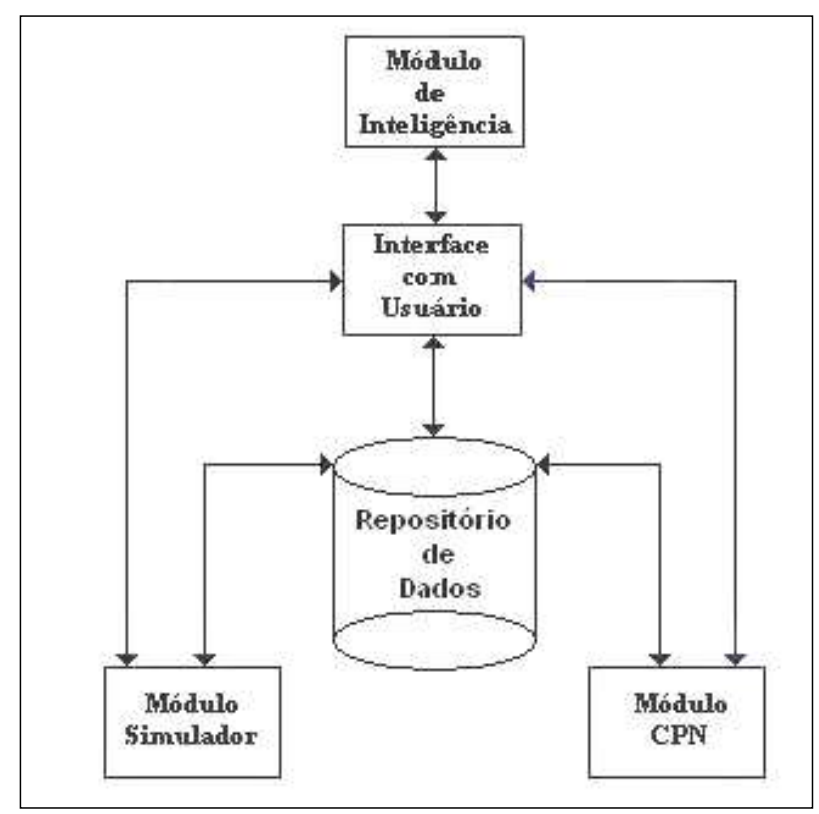

Figura 1: Arquitetura do SAD

O funcionamento da arquitetura proposta é resumido a seguir. O módulo de interface, a partir de parâmetros recebidos do usuário, gera uma série de arquivos que são gravados no repositório de dados. Estes arquivos têm como objetivo disponibilizar os parâmetros de entrada selecionados e criar a lógica que o módulo simulador deverá seguir durante a simulação. Durante a seleção destes parâmetros o auxílio do módulo de inteligência pode ser requisitado para que os significados dos dados fornecidos nesta interface sejam compreendidos e correspondam às respostas esperadas pelo usuário em relação ao cenário que se deseja representar. Definidos estes dados, o módulo simulador é inicializado através de um comando na interface de entrada. Após, o módulo simulador busca os dados que necessita no repositório de dados e configura qual a lógica que o programa de simulação irá adotar em relação ao processo. Também, para cada etapa do processo representada no modelo, existe um conjunto de distribuições estatísticas baseadas na coleta de dados do processo que se deseja analisar. Assim, a simulação é iniciada e o usuário do SAD pode acompanhar, em uma interface gráfica, a execução durante o período de tempo selecionado para simulação do processo. Durante a simulação, os dados resultantes são gravados em arquivos no módulo repositório de dados. Concluída a simulação, o módulo de interface com o usuário busca os resultados nos arquivos do repositório, manipula alguns dados através de cálculos simples e exibe-os na interface de resultados. Para que este conjunto de soluções seja interpretado com maior facilidade o módulo de inteligência novamente pode ser acionado, para que através da interação com o SE as conclusões consideradas corretas sejam alcançadas pelo usuário. Os resultados são exibidos para o usuário e cabe a este interpretar se o cenário em avaliação 
corresponde aos resultados almejados, ou se existe alguma solução mais eficiente testada anteriormente armazenada no histórico de simulações $(\log )$. Já o módulo CPN que valida estruturalmente o modelo proposto no simulador, independe dos outros módulos e pode ser utilizado para qualquer cenário. Seus resultados são armazenados no repositório de dados para posterior análise. Mais alguns detalhes dos principais módulos do sistema serão vistos adiante.

Especificamente, através da seleção de alguns parâmetros o gestor pode obter os seguintes resultados:

- Quantidade Produzida (quantidade total de peças que são concluídas);

- Produção Diária (produção diária atingida considerando o período de simulação selecionado);

- WIP (média total da quantidade de peças no processo de fabricação "inteiro");

- Gráfico com o estado das entidades (em operação, bloqueadas ou aguardando recursos);

- Gráfico com utilização dos recursos (humanos e máquinas);

- Gráfico de utilização das etapas (que no modelo desenvolvido representam as etapas de fabricação);

- Gráfico com estado das etapas (operation, idle, waiting, bloqued, down);

- Gráfico com estado dos recursos (ocupados ou livres);

- Relação dos recursos (humanos e máquina) que estão sendo utilizados mais de $60 \%$ do tempo;

- Contadores de tempo de permanência em cada setor, em porcentagem, mediante o processo produtivo (para identificar gargalos por setor);

- Possibilidade de consultar documentação com dados do processo (coleta de tempos de produção por etapa, siglas dos recursos máquina e postos de trabalho humanos, etapas do processo em ordem de fabricação indicando postos possíveis e recursos utilizados em cada uma delas, distribuições estatísticas utilizadas).

\subsection{Interface com o Usuário}

Este módulo divide-se em duas partes chamadas de: Módulo Gestor - Entrada de Dados e Módulo Gestor - Resultados. Utilizando a Interface de Entrada de Dados o gestor seleciona e altera os parâmetros desejados, antes de executar a simulação. Após o término desta seleção, estes parâmetros são salvos em arquivos texto no Repositório de Dados e a simulação é iniciada. Concluída esta etapa, os resultados são exibidos ao usuário através da interface de Resultados e novamente armazenados em arquivos no Repositório, mantendo o histórico de utilização do SAD.

\subsection{Módulo de Inteligência}

É constituído do Sistema Especialista que foi desenvolvido com objetivo de auxiliar o gestor na utilização do SAD. Este sistema está dividido em dois módulos que estão acessíveis a partir das interfaces que constituem o Módulo de Interface com o Usuário. O primeiro módulo auxilia na definição dos dados que devem ser fornecidos na Interface de Entrada de Dados e o segundo na interpretação dos resultados exibidos na Interface de Resultados. Basicamente a função do SE é representar a base de conhecimento elaborada no momento da criação do SAD, para auxiliar na entrada dos dados do sistema e interpretação dos resultados que são apresentados. Isto simplifica a utilização pois o usuário do SAD (gestor) não precisa preocupar-se em relacionar os dados que foram fornecidos ou alterados no $\mathrm{SAD}$ e quais as conseqüências que estas alterações provocaram nos resultados.

A estrutura do SE pode ser decomposta nas seguintes partes:

- Objetivos: conjunto de atributos das variáveis resultantes para qual o auxílio do SE foi solicitado (auxílio na entrada de dados ou interpretação dos resultados);

- Variáveis: divididas em variáveis objetivo e atributos. As variáveis objetivo do sistema são compostas de diversos atributos que formalizam o diagnóstico apresentado pelo SE;

- Interface: opção onde são inseridas as perguntas referentes às variáveis (quando existir);

- Regras: condições criadas que representam a base de conhecimento.

Pode-se apresentar uma das regras como exemplo:

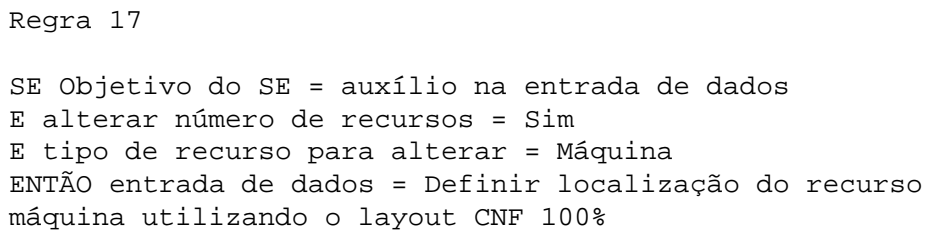

Se as três condições iniciais são satisfeitas o diagnóstico relacionado na variável objetivo "entrada de dados" é apresentada com grau de confiança de $100 \%$. As regras que formam o SE são relacionadas através da máquina de inferência 
da shell, que está fundamentada no encadeamento para trás (backward chaining). Conforme (Lia, 2003), o Expert Sinta utiliza um modelo de representação de conhecimento basedo em regras de produção e fatores de confiança.

\subsection{Repositório de Dados}

Tem como objetivo armazenar todas as informações que são fornecidas no módulo de Interface com o Usuário, os resultados obtidos pelo Simulador e CPNs, e todos os dados utilizados pelo Simulador. Este repositório também armazena o conjunto de logs dos diversos cenários avaliados. O gestor terá os parâmetros que selecionou na Interface de Entrada de Dados (antes de rodar a simulação) e os resultados que ele obteve na Interface de Resultados, gravados neste log, indexados pela data e hora da simulação. Além de servir para futuras avaliações, o maior objetivo desta base é possibilitar que os diferentes cenários de testes desenvolvidos sejam comparados, sem que haja necessidade da simulação ser repetida.

\subsection{Módulo Simulador}

Este módulo contém o modelo do processo de fabricação, onde foram agregados os dados coletados durante o mapeamento do processo: etapas de fabricação, seqüência de etapas, recursos humanos, recursos máquina, tempos de fabricação, entre outros que estão inseridos através de funções e elementos típicos utilizados em simulação. Neste módulo também são processadas as requisições feitas pelo Módulo de Interface com o Usuário, de onde são originados os parâmetros enviados ao simulador e destinados os resultados após a simulação. Importantes elementos deste módulo estão descritos no item 4.3, já que são vinculados fortemente à implementação.

\subsection{Módulo CPN}

Este módulo é constituído pela mesma estrutura representada no Simulador e, na versão atual, tem como objetivo complementar os resultados obtidos com o simulador e verificar se o modelo do processo é estruturalmente válido. A filosofia de construção do modelo adotada foi a criação de uma estrutura hierárquica do tipo top-down com fusion places (Jensen, 1996). Apesar das inúmeras propriedades existentes nesta técnica, neste trabalho foi considerada apenas a verificação de deadlock que é considerada relevante em processos industriais, segundo (Dicesare et alli, 1993). Similarmente ao Módulo simulador, importantes elementos deste módulo estão descritos no item 4.4, já que são fortemente vinculados à implementação.

\section{IMPLEMENTAÇÃO}

O diagrama apresentado na Figura 3 tem como objetivo auxiliar na interpretação de como o usuário interage com os módulos do protótipo e como estes interagem entre si, identificando qual o software utilizado em cada um deles.

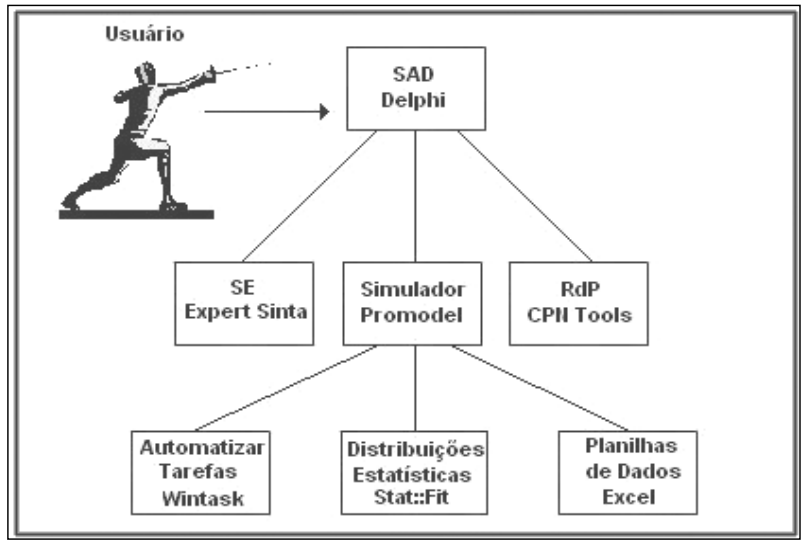

Figura 3: Relacionamento do Usuário com os Módulos

\subsection{Interface}

Conforme visto, o módulo de Interface com o Usuário está dividido em duas telas chamadas de Módulo Gestor - Entrada de Dados e Módulo Gestor - Resultados. As Figuras 4 e 5 ilustram, respectivamente, estas telas. As interfaces de ambas as telas foram criadas utilizando as ferramentas gráficas disponíveis na linguagem Delphi, que facilmente são inseridas nos formulários e auxiliam na programação das funções, associadas a cada botão. Basicamente as opções escrevem valores em arquivos texto de onde o Simulador lê estas informações e define qual a lógica que será utilizada durante a simulação. Para exemplificar uma das opções desta interface será explicada. Para selecionar o roteiro dos pedidos, o gestor pode optar pela simulação da produção completa, maquete e matriz, ou somente pela produção da matriz. No momento que é selecionada uma das opções o programa escreve um valor numérico em um arquivo texto que é lido posteriormente pelo Simulador. Sendo assim, dependendo deste valor, o simulador saberá qual o roteiro que deverá simular (completo ou somente parte dele). Da mesma forma o usuário seleciona a maneira com que os recursos serão alocados, o número de pedidos que serão simulados, o período de simulação, entre outros.

Já para o Módulo Gestor - Resultados basicamente todos os valores são lidos de arquivos texto onde o simulador armazena os dados que obteve como resultado, que são diretamente dependentes dos parâmetros de entrada. O layout foi definido para apresentar todos estes resultados de maneira simples, para que o gestor pudesse facilmente interpretar seu 


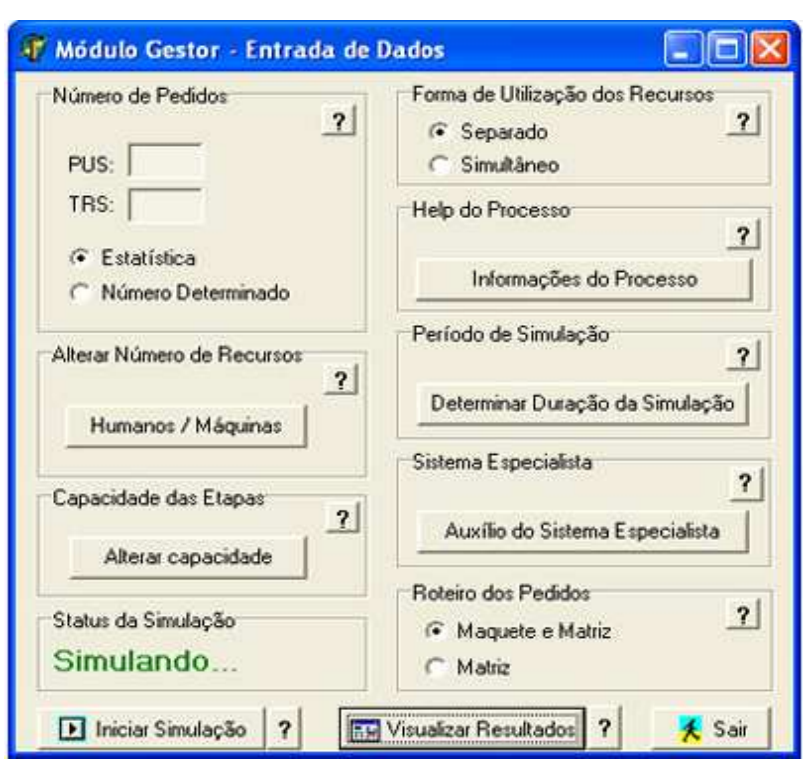

Figura 4: Tela do Módulo Gestor - Entrada de Dados

significado, conforme a Figura 5. Neste caso, o gestor optou que fossem listados os recursos que tivessem mais de $60 \%$ do tempo ocupado realizando alguma atividade do processo.

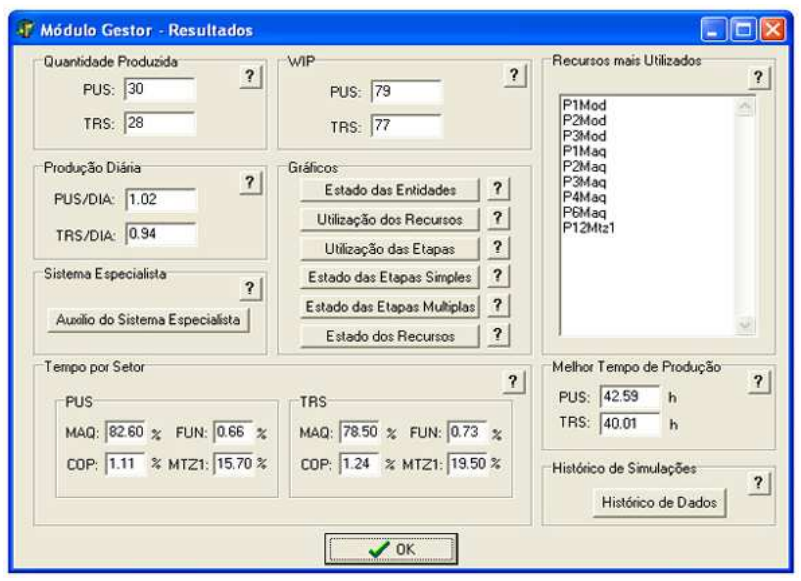

Figura 5: Tela do Módulo Gestor - Resultados

\subsection{Módulo de Inteligência}

O SE foi criado utilizando a shell Expert Sinta, a partir de uma base de conhecimento construída durante a fase de coleta de dados e estudo do processo. No próximo passo foram determinadas as variáveis objetivo do SE, as quais possuem uma série de outras variáveis associadas que compõem o conjunto de resultados possíveis apresentados, de acordo com a aprovação das regras, no diagnóstico de saída. A Figura 6 mostra, a título de exemplo, um conjunto de resultados obtidos pelo usuário ao solicitar auxílio do SE.

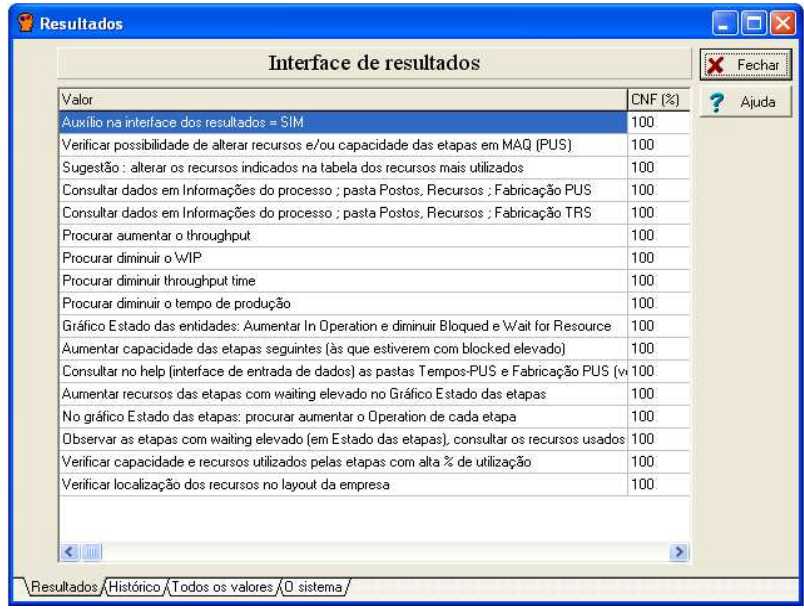

Figura 6: Resultados Apresentados pelo SE

\subsection{Módulo Simulador}

A Figura 7 mostra um pequeno esboço da metodologia utilizada no modelo desenvolvido no Promodel, onde foram definidos os principais elementos do modelo:

- Locations: representam as etapas do processo produtivo de produção de matrizes;

- Conveyor: representam os "armazenadores" de peças entre as etapas de produção;

- Resources: representam os recursos humanos e máquinas/ferramentas do processo;

- Entities: representam as peças que estão sendo produzidas;

- Processing: programação que é executada em cada etapa e definições para onde a peça deve seguir (roteamento entre etapas);

- Arrivals: distribuições estatísticas que definem a entrada de pedidos no modelo;

- Shifts: determinam os períodos do dia (horários) em que a empresa está produzindo.

Para o caso estudado este modelo possui 95 etapas de processo, onde cada uma delas foi representada por uma location para onde os recursos são alocados à medida que são necessários. Isto torna o modelo bastante versátil para alterações de parâmetros como: seqüência produtiva, etapas, recursos utilizados e distribuições estatísticas que representam os tempos de processo. Porém, a definição estrutural utilizada difere-se das usuais que utilizam path networks para definir o caminho que as peças que estão sendo produzidas 


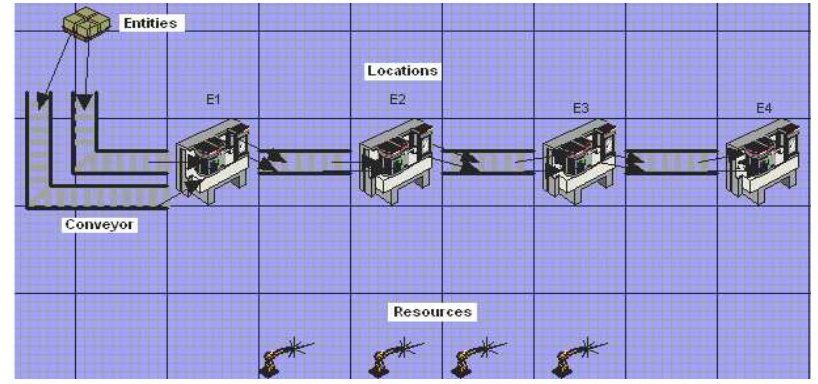

Figura 7: Componentes Utilizados no Modelo de simulação

devem seguir. Isto devido a grande quantidade de etapas e postos de trabalho no processo. O conceito de posto de trabalho foi abstraído em etapas, independente de quantos existirem, sendo os recursos humanos/máquinas ou peças que compõem o produto são alocados em cada etapa, à medida que forem necessários. É importante salientar que as etapas possuem capacidade igual ao número de recursos humanos que sabem executá-la. Com isto simplificou-se a representação estrutural e gráfica do modelo facilitando alterações conforme pode ser comprovado em (Hennemann, 2004), garantindo ao usuário a coerência do modelo com relação ao processo real (transparência). Concluídas as etapas de formulação do problema, coleta de dados e definição da estrutura do modelo, conforme (Law e Kelton, 2000), sugere-se que seja feita a primeira validação. Além da estrutura foram verificadas se as distribuições estatísticas geradas para simular os tempos das etapas de processo estariam coerentes. Foram utilizados testes estatísticos de Kolmogorov Smirnov e Anderson Darling, que conforme (Law e Kelton, 2000) e (Banks et alli, 1996) são adequados para pequenas amostras e os resultados foram adequados.

\subsection{Módulo CPN}

A Figura 8 representa a estrutura hierárquica da rede desenvolvida com o auxílio do software CPNTools.

A complexidade do modelo CPN pode ser distribuída nas diversas partes que compõem uma rede, ou seja, nas inscrições, declarações e na própria estrutura. A estrutura é constituída por lugares, transições e arcos como nas redes PT (PlaceTransition) convencionais. Já as declarações definem os conjuntos de cores (domínios), variáveis e funções usadas nas inscrições. Após criar a estrutura da rede, seguindo a mesma lógica utilizada no modelo desenvolvido no módulo simulador, foram criadas as subpáginas que representam os setores. Por exemplo, na Figura 6 existe uma sub-página que representa a entrada dos pedidos, através da transição Pedidos no setor de maquetaria, representado pelo lugar denominado MAQ. Logo, os pedidos entram pela sub-página representada pela transição Pedidos, são encaminhados ao setor de

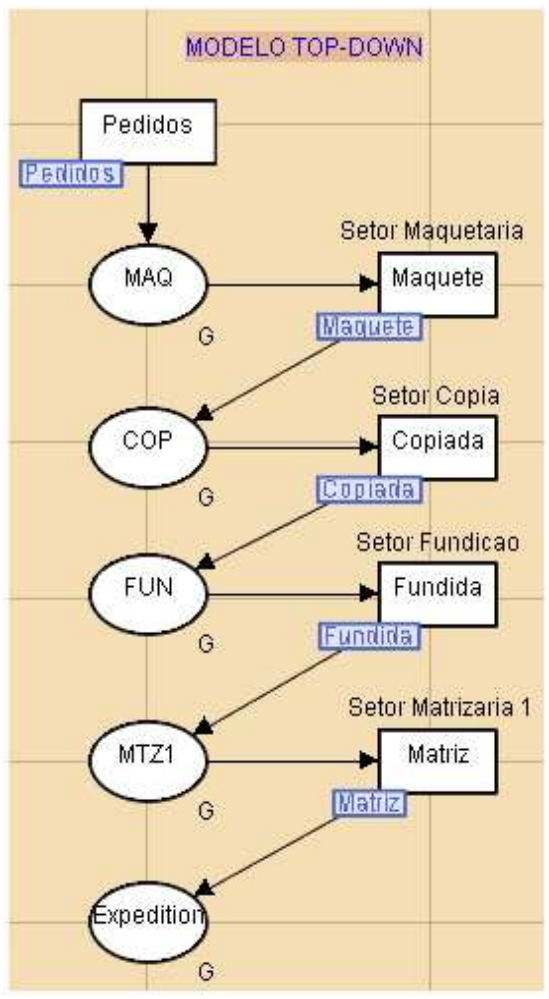

Figura 8: Estrutura Hierárquica

maquetaria no lugar MAQ que por sua vez possui outra subpágina vinculada representada pela transição Maquete. Nesta sub-página todas as etapas do setor de maquetaria estão modeladas. Quando estas etapas estiverem concluídas o pedido é encaminhado ao setor de Cópia, representado pelo lugar COP e assim sucessivamente, até percorrer todos os setores e, conseqüentemente, todas as etapas de fabricação. As declarações e inscrições foram programadas de acordo com a seqüência de etapas, recursos necessários em cada uma delas e as distribuições estatísticas representando os tempos de processo. A Figura 9 representa parte do modelo desenvolvido, a título de exemplo.

A CPN acima representa a alocação de cada recurso, o tempo gasto em cada etapa e a devolução deste, auxiliada pelas funções guarda e inscrições dos arcos. Pode-se citar um exemplo de representação na rede referente aos recursos humanos do processo. No lugar denominado PMaq, existem 22 pessoas representadas por um único lugar. Cada uma delas está habilitada a executar um conjunto de etapas distintas. Com a função guarda é possível selecionar quem está habilitado a executar cada etapa. As demais configurações referentes às cores dos lugares, valores das variáveis utilizadas na rede e representação das funções estatísticas são programas externamente no conjunto de declarações. Sem estas propriedades de atribuir características individuais através de conjuntos de 


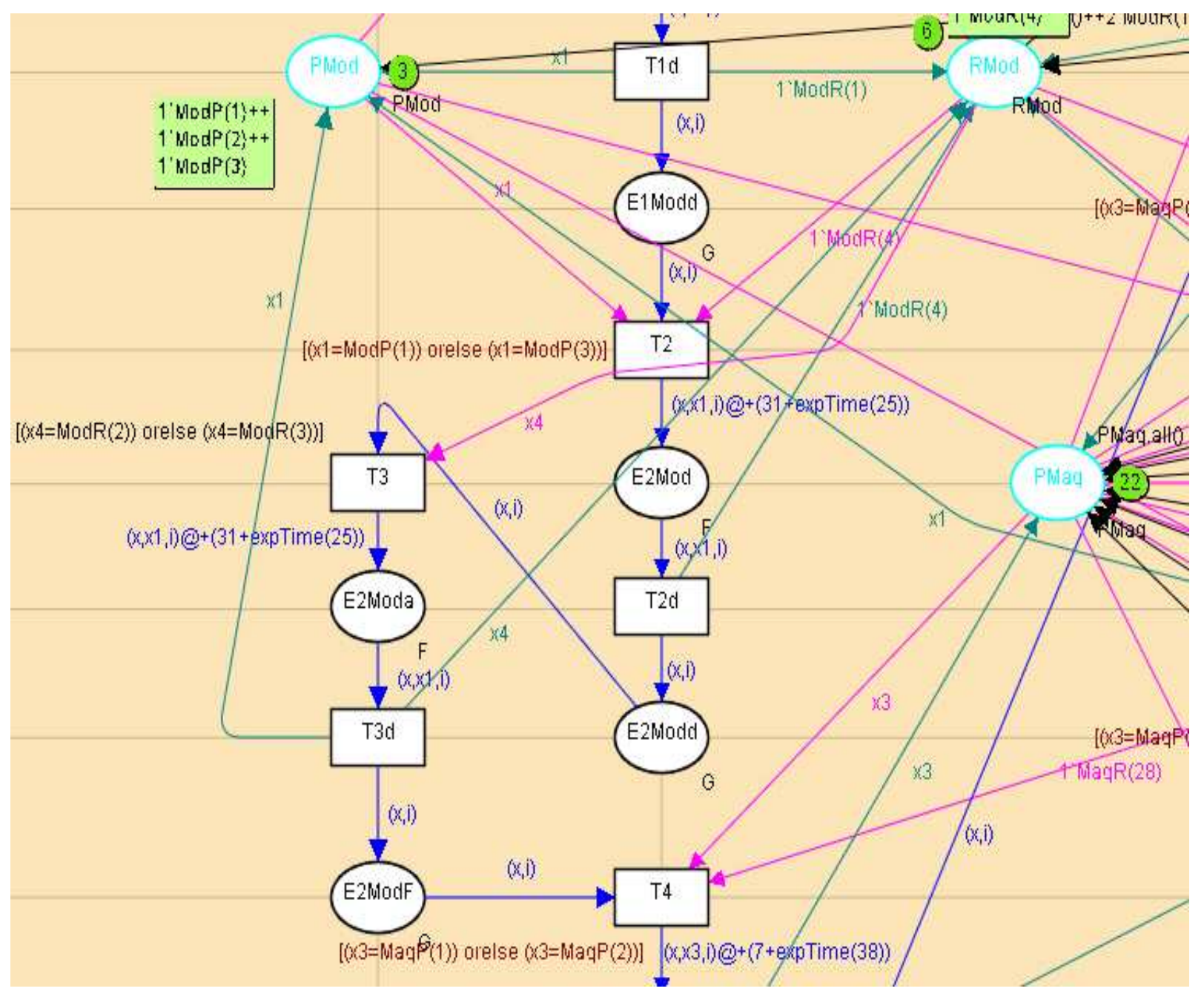

Figura 9: Exemplo do Modelo Desenvolvido com CPN

cores, somente para este exemplo, em redes PT teríamos 22 lugares, com 22 nomes diferentes e não poderíamos colocar a condição OU selecionando uma ou outra pessoa. Por este e outros motivos através de Redes de Petri Convencionais ficaria praticamente inviável representar a complexidade do processo ao qual se está tratando neste trabalho.

\section{TESTES E RESULTADOS}

\subsection{Sistema produtivo real}

Com objetivo de avaliar e validar o SAD proposto, dados reais de um processo de fabricação foram coletados em uma empresa que produz maquetes e matrizes no segmento de calçados. Como o protótipo é genérico, foram parametrizadas as variáveis de entrada e saída juntamente com os gestores que seriam os usuários do SAD. Primeiramente foi criada uma nomenclatura padrão para tratar estes dados. Para recursos, $\mathrm{P}$ indica pessoa e $\mathrm{R}$ indica máquina. A primeira letra é seguida de um número que identifica o recurso e finaliza com 3 letras que correspondem ao setor. Exemplo: P1Mod (recurso pessoa, número 1, que trabalha no setor de Modelagem). Além disto foram classificados os tipos de produtos, aos quais a empresa produz, para que através do mapeamento de etapas de produção, postos de trabalho e recursos necessários, fosse possível construir o modelo do processo que corresponde a um dos módulos do SAD. Nos cenários avaliados neste trabalho foram citados os dois produtos principais da empresa, ou seja, PUS e TRS.

\subsection{Cenário 1}

Utilizando o SAD para avaliar o que se classificou como cenário atual (sem modificações de recursos) observou-se que a Maquetaria corresponde ao maior percentual em tempo de processo, considerando todas as etapas de produção. A par- 
tir da Tabela 1 foram observados os recursos com maior índice de utilização, na maquetaria, para a matriz do tipo PUS. Como todos estes recursos são humanos, sugere-se o treinamento de mais pessoas para desempenhar as etapas correspondentes. Isto não implica em novas contratações, mas sim o treinamento de pessoas ociosas para desempenhar novas atividades. Este critério de escolha pode basear-se nos gráficos de tempo de utilização de recursos, também disponibilizados pelo SAD.

\begin{tabular}{|l|c|}
\hline \multicolumn{2}{|c|}{ PUS (Maquete) } \\
\hline Quantidade Produzida & 32 \\
\hline PUS/Dia & 1,15 \\
\hline WIP & 83 \\
\hline Recursos mais utilizados & P1Mod, P2Mod,P3Mod \\
\hline Recursos mais utilizados & P1Maq,P2Maq,P3Maq,P4Maq \\
\hline Em operação & $59,25 \%$ \\
\hline Bloqueado & $26,60 \%$ \\
\hline Aguardando recursos & $14,13 \%$ \\
\hline
\end{tabular}

Tabela 1: Cenário Atual

Após duplicação dos recursos humanos indicados na Tabela 1 os novos resultados são apresentados na Tabela 2.

\begin{tabular}{|l|c|}
\hline \multicolumn{2}{|c|}{ PUS (Maquete) } \\
\hline Quantidade Produzida & 50 \\
\hline PUSIDia & 1,73 \\
\hline WIP & 88 \\
\hline Recursos mais utilizados & P1Mod, P2Mod,P3Mod,R1Mod \\
\hline Recursos mais utilizados & R4Mod, P1Maq,P2Maq,P3Maq \\
\hline Recursos mais utilizados & P4Maq,P22Maq \\
\hline Em operaçäo & $60,77 \%$ \\
\hline Bloqueado & $25,08 \%$ \\
\hline Aguardando recursos & $14,13 \%$ \\
\hline
\end{tabular}

Tabela 2: Cenário 1

Observou-se que a mudança sugerida trouxe benefícios com relação ao cenário atual:

- Houve aumento de produtividade de 56\% para as maquetes do tipo PUS;

- A produção diária aumentou de 1,15 para 1,73 ;

- O tempo em que o produto fica em operação aumentou (produto em um posto de trabalho sendo processado);

- O tempo de sistema bloqueado diminuiu (tempo de espera do produto antes de iniciar a etapa seguinte);

- Considerando o processo completo (maquete e matriz), cujos dados não estão nas tabelas acima, o produto do tipo PUS teve aumento de produção de $58 \%$.
Conclui-se que o cenário 1 proposto introduz melhorias ao cenário atual, principalmente em relação ao acréscimo significativo de produção no setor de maquetaria, que nitidamente se mostra como o gargalo do processo. Com este aumento de produtividade, com baixo investimento em capacitação, a empresa poderia reduzir as terceirizações dos atuais $50 \%$ para $25 \%$, aproximadamente.

\subsection{Cenário 2}

Visando aprimorar os resultados encontrados nas simulações do cenário 1, as alterações sugeridas na Tabela 3 serão realizadas utilizando o SAD. Observa-se que a maioria das alterações são capacitação de pessoas, similar ao cenário 1 .

\begin{tabular}{|c|c|}
\hline Recursos anteriores & Recursos Modificados \\
\hline 1 P1Mod & 3 P1Mod \\
\hline 1 P2Mod & 3 P2Mod \\
\hline 1 P3Mod & 3 P3Mod \\
\hline 1 P1Maq & 3 P1Maq \\
\hline 1 P2Maq & 3 P2Maq \\
\hline 1 P3Maq & 3 P3Maq \\
\hline 1 P4Maq & 3 P4Maq \\
\hline 1 P22Maq & 2 P22Maq \\
\hline 3 R1Mod & 5 R1Mod \\
\hline 1 R4Mod & 2 R4Mod \\
\hline
\end{tabular}

Tabela 3: Alterações no Cenário 1

Estas alterações estão baseadas nos recursos que apresentaram o maior índice de utilização nas simulações feitas para o cenário 1. Os resultados encontrados estão listados na Tabela 4.

\begin{tabular}{|l|c|}
\hline \multicolumn{2}{|c|}{ PUS (Maquete e Matriz) } \\
\hline Quantidade Produzida & 71 \\
\hline PUS/Dia & 2,42 \\
\hline WIP & 97 \\
\hline Recursos mais utilizados & P1Mod, P2Mod,P3Mod,R1Mod \\
\hline Recursos mais utilizados & R2Mod, R4Mod,P1Maq,P2Maq \\
\hline Recursos mais utilizados & P3Maq,P4Maq,P6Maq,P7Maq \\
\hline Recursos mais utilizados & P8Maq,P9Maq,P10Maq,P13Maq \\
\hline Em operação & $57,90 \%$ \\
\hline Bloqueado & $28,24 \%$ \\
\hline Aguardando recursos & $13,85 \%$ \\
\hline
\end{tabular}

Tabela 4: Cenário 2

Comparando-se os resultados do cenário 2 com o cenário atual (tabela 1), destacam-se os seguintes resultados:

- Houve aumento de produtividade de mais de $100 \%$ de maquetes do tipo PUS; 
- A produção diária aumentou de 1,15 para 2,42;

- O tempo de espera por disponibilidade de recursos diminuiu de 14,13 para $13,85 \%$;

- A produção de maquete e matriz também aumentou mais de $100 \%$ para PUS;

Observou-se que a quantidade de recursos com maior tempo de ocupação aumentou consideravelmente. Isto indica que a distribuição de tarefas entre as pessoas deste setor está mais homogênea, fato comprovado pelo aumento considerável de produção.

\subsection{Cenário 3}

Os cenários 1 e 2 tiveram como objetivo tratar o gargalo do processo, ou seja, a maquetaria. Como o resultado obtido foi significativo, os cenários 3 e 4 serão utilizados para avaliar todas as etapas de produção que incluem a maquete e matriz do calçado. A Tabela 5 mostra os resultados apresentados pelo $\mathrm{SAD}$, para o cenário atual de produção de maquete e matriz (processo completo) do tipo PUS. Similarmente a Tabela 6 apresenta estes dados para maquete e matriz do tipo TRS.

\begin{tabular}{|l|c|}
\hline \multicolumn{2}{|c|}{ PUS (Maquete e Matriz) } \\
\hline Quantidade Produzida & 31 \\
\hline PUS/Dia & 1,03 \\
\hline WIP & 78 \\
\hline Recursos mais utilizados & P1Mod, P2Mod,P3Mod \\
\hline Recursos mais utilizados & P1Maq, P2Maq,P3Maq,P4Maq \\
\hline Em operação & $58,05 \%$ \\
\hline Bloqueado & $25,71 \%$ \\
\hline Aguardando recursos & $16,22 \%$ \\
\hline
\end{tabular}

Tabela 5: Cenário atual para PUS

\begin{tabular}{|l|c|}
\hline \multicolumn{2}{|c|}{ TRS (Maquete e Matriz) } \\
\hline Quantidade Produzida & 34 \\
\hline PUS/Dia & 1,15 \\
\hline WIP & 78 \\
\hline Recursos mais utilizados & P1Mod, P2Mod,P3Mod \\
\hline Recursos mais utilizados & P1Maq, P2Maq,P3Maq,P4Maq \\
\hline Em operação & $63,34 \%$ \\
\hline Bloqueado & $23,85 \%$ \\
\hline Aguardando recursos & $12,79 \%$ \\
\hline
\end{tabular}

Tabela 6: Cenário atual para TRS

Seguindo a mesma lógica utilizada nos cenários anteriores, os recursos que apareceram como mais utilizados nas tabelas 5 e 6 foram duplicados, já que os mesmos implicam apenas em investimentos de capacitação de recursos humanos. Os resultados obtidos foram listados na Tabela 7 e 8.

\begin{tabular}{|l|c|}
\hline \multicolumn{2}{|c|}{ PUS (Maquete e Matriz) } \\
\hline Quantidade Produzida & 49 \\
\hline PUS/Dia & 1,66 \\
\hline WP & 86 \\
\hline Recursos mais utilizados & P1Mod, P2Mod,P3Mod,R1Mod,R4Mod \\
\hline Recursos mais utilizados & P1Maq, P2Maq,P3Maq,P4Maq \\
\hline Recursos mais utilizados & R4Mod, P22Maq \\
\hline Em operação & $60,51 \%$ \\
\hline Bloqueado & $24,66 \%$ \\
\hline Aguardando recursos & $14,81 \%$ \\
\hline
\end{tabular}

Tabela 7: Modificações no Cenário 3 para PUS

\begin{tabular}{|l|c|}
\hline \multicolumn{2}{|c|}{ TRS (Maquete e Matriz) } \\
\hline Quantidade Produzida & 50 \\
\hline PUS/Dia & 1,68 \\
\hline WIP & 86 \\
\hline Recursos mais utilizados & P1Mod, P2Mod,P3Mod,R1Mod,R4Mod \\
\hline Recursos mais utilizados & P1Maq, P2Maq,P3Maq,P4Maq \\
\hline Recursos mais utilizados & R4Mod, P22Maq \\
\hline Em operação & $59,01 \%$ \\
\hline Bloqueado & $24,27 \%$ \\
\hline Aguardando recursos & $16,71 \%$ \\
\hline
\end{tabular}

Tabela 8: Modificações no Cenário 3 para TRS

Algumas observações podem ser feitas com relação aos resultados obtidos com o cenário 3 :

- A produtividade de maquetes e matrizes (produção completa) de PUS aumentou 58\% e TRS aumentou $47 \%$;

- A produção diária para PUS e TRS aumentou;

- Em PUS o tempo de produto em operação aumentou e o tempo de produto bloqueado e aguardando recursos diminuiu, o que justifica um percentual maior de produtividade se comparado a TRS;

- TRS foi mais afetada pelo aumento na utilização dos recursos pois seu percentual de tempo em operação diminuiu e o de espera por recursos aumentou.

Conclui-se que o cenário 3 proposto possui características mais adequadas que o cenário atual, apenas investindo em capacitação de funcionários para executar mais etapas. Apesar do aumento de recursos humanos, os recursos que ainda aparecem com alta utilização são da modelagem, o que comprova que o gargalo do processo encontra-se nas etapas iniciais do processo, ou seja, na produção de maquetes. 


\subsection{Cenário 4}

No cenário 4 os recursos humanos que apareceram como mais utilizados no cenário 3 foram triplicados (apenas capacitação)., e os recursos máquina duplicados. Os resultados obtidos estão listados na Tabela 9 e 10.

\begin{tabular}{|l|c|}
\hline \multicolumn{2}{|c|}{ PUS (Maquete e Matriz) } \\
\hline Quantidade Produzida & 66 \\
\hline PUS/Dia & 2,21 \\
\hline WP & 89 \\
\hline Recursos mais utilizados & P1Mod, P2Mod,P3Mod,R1Mod, R2Mod \\
\hline Recursos mais utilizados & P1Maq, P2Maq,P3Maq,P4Maq,PSMaq \\
\hline Recursos mais utilizados & P6Maq, P7Maq, P8Maq,P9Maq,P21Maq \\
\hline Em operação & $52,45 \%$ \\
\hline Bloqueado & $27,61 \%$ \\
\hline Aguardando recursos & $19,93 \%$ \\
\hline
\end{tabular}

Tabela 9: Modificações no Cenário 4 para PUS

\begin{tabular}{|l|c|}
\hline \multicolumn{2}{|c|}{ TRS (Maquete e Matriz) } \\
\hline Quantidade Produzida & 65 \\
\hline PUS/Dia & 2,17 \\
\hline WIP & 115 \\
\hline Recursos mais utilizados & P1Mod, P2Mod,P3Mod,R1Mod, R2Mod \\
\hline Recursos mais utilizados & P1Maq, P2Maq,P3Maq,P4Maq,PMaq \\
\hline Recursos mais utilizados & P6Maq, P7Maq, P8Maq,PM Maq,P21Maq \\
\hline Em operação & $55,31 \%$ \\
\hline Bloqueado & $24,55 \%$ \\
\hline Aguardando recursos & $20,12 \%$ \\
\hline
\end{tabular}

Tabela 10: Modificações no Cenário 4 para TRS

Comparando os resultados obtidos para o cenário 4 , com os dados do cenário atual do cenário 3 (tabelas 5 e 6), conclui-se que:

- A produtividade de maquetes e matrizes (produção completa) aumentou em mais de 100\% para PUS e TRS;

- A produção diária de PUS e TRS aumentou;

- O tempo em que PUS e TRS estão em operação diminuiu e o tempo que aguardam recursos aumentou;

- Apareceram vários recursos, que não estavam na lista anterior, com alto índice de utilização.

Conclui-se que, apesar dos resultados do cenário 4 terem sido significativos, deve-se observar que nem sempre todos os parâmetros melhoram nas mudanças de cenário. Este é um dos objetivos do SAD, pois os parâmetros exibidos como resultado ao gestor foram escolhidos por ele mesmo no momento do projeto do sistema e servem de subsídios para tomada de decisão.
Considerando-se a empresa estudada através dos cenários propostos, observa-se claramente que o gargalo do processo produtivo está nas etapas iniciais, ou seja, na produção de maquetes. Os recursos relacionados com estas etapas apareceram com alto índice de utilização em todos os cenários testados. Mas, o aumento de produção e o aumento de recursos ocupados provaram que o efeito do gargalo foi reduzido, havendo melhorias na distribuição da produção entre as etapas de processo. Com pequenos investimentos em capacitação do quadro funcional a produção dobrou. Mesmo assim, os recursos que ainda aparecem com alto índice de utilização ainda são na maioria humanos, o que sugere que o problema principal deste processo é capacitar as pessoas para que estas saibam executar um maior número de etapas.

\subsection{Validação Estrutural}

O primeiro modelo de CPNs desenvolvido mostrou que a metodologia de alocação de recursos de forma simultânea, onde todos os recursos são buscados pela etapa (conjunto completo) não é apropriada para representar o processo produtivo pois, dos 390 estados do diagrama de Espaço de Estados gerado pelo CPN Tools, foram encontrados 256 estados possíveis de deadlock. Já para o modelo desenvolvido que aloca os recursos individualmente (opção separada do SAD), que representa o funcionamento normal do processo, foi criado outro modelo usando CPNs, para o qual também foi calculado o espaço de estados. Neste foram encontrados apenas dois estados possíveis da rede entrar em deadlock. Isto comprova que esta metodologia de alocação de recursos é mais apropriada para o modelo desenvolvido neste processo e, portanto, apropriada para validar o sistema e ser utilizada no $\mathrm{SAD}$. Para o segundo cenário proposto também foram encontrados dois estados possíveis de deadlock, o que demonstra que, apesar de superar o cenário atual, ainda não é a distribuição mais adequada para o processo em avaliação. Da mesma forma, cada cenário proposto poderá ser avaliado utilizando esta técnica com o objetivo de encontrar cenários sem nenhuma ocorrência de deadlocks, assegurando, com isto, que o sistema modelado poderá produzir independentemente do estado que se encontre. Apesar desta técnica complementar os resultados obtidos pelo simulador, o modelo mostrou-se com complexidade elevada para proporcionar respostas rápidas para o SAD.

\subsection{Decorrências dos resultados}

Através da análise dos cenários propostos, foi possível obter uma série de conclusões sobre o processo produtivo de maquetes e matrizes. Algumas se destacam e serão relacionadas, genericamente, como sugestões para melhorar o desempenho do processo produtivo de fabricação deste tipo de produto. Segue a relação de sugestões: 
Conforme testes realizados nos cenários um aumento de recursos humanos trouxe ganhos significativos para o setor de maquetaria. Isto sugere que se houvesse um programa de capacitação de funcionários que possuem um baixo índice de utilização (que pode ser verificado no gráfico de utilização de recursos) para executar as mesmas etapas que os recursos humanos com alta porcentagem de utilização executam, poderia haver um equilíbrio de utilização de recursos e um ganho potencial de produção, conforme foi verificado nos cenários propostos;

- Através de pequenas alterações no setor de maquetaria a empresa poderia ficar independente da prestação de serviços de terceirizados;

- Equilibrando a utilização dos recursos a empresa poderia optar pela descontinuidade do expediente noturno que, apesar de poucas etapas e pessoas, demanda gastos com recursos. Estes gastos poderiam implicar em aumento de produção ou economia de recursos;

- Para aumentar a produtividade do processo completo ou de maquetes apenas, os recursos identificados como de maior gargalo no cenário 1 devem ser equilibrados em sua utilização. Além disto, com o aumento destes (em número) surgem vários outros com altas taxas de utilização que devem ser também equilibrados. Para o caso da maquetaria, maior gargalo do processo completo, sugere-se um programa intensivo de capacitação dos próprios funcionários para desempenhar mais etapas do processo. O SAD pode auxiliar nos testes de índices de produtividade almejados, à medida que os recursos humanos estiverem capacitados para exercerem mais etapas;

- A implantação de um programa de qualidade no processo poderia evitar a alta taxa de retrabalho atual pois ficou constatado que a empresa pode atingir seus objetivos no horário de funcionamento normal (diurno), sem necessitar de hora extra e nem expediente noturno;

- Os eventuais investimentos em recursos máquina (ou até humanos) devem ser testados com auxílio do SAD para que se verifique o resultado real antes de implementar as alterações. Ficou comprovado que a ampliação de recursos não implica, necessariamente, em resultados significativos para a matrizaria;

- Elaborar um estudo da frequiência com que os recursos apresentam problema para verificar a necessidade de colocar equipamentos sobressalentes nos pontos mais críticos do processo. Verifica-se hoje que não existe plano de contingência para estas situações e o SAD pode auxiliar na previsão dos transtornos que poderiam ser causados pela falta de recursos considerados essenciais.

\subsection{Comparação entre simulação e CPNs}

Comparando-se as duas técnicas de modelagem que, em suma, possuem os mesmos potenciais quanto à modelagem $\mathrm{e}$ simulação de processos conclui-se que:

- A interface do simulador Promodel facilitou a representação do processo real de fabricação em um modelo, devido à complexidade do mesmo. Já para CPNs, apesar do nível de abstração, a representação ficou bastante complexa;

- O Algoritmo construtivo do modelo no Promodel é mais simples de ser programado do que em CPNs;

- Utilizando o Promodel obtinha-se a simulação de meses de fabricação em apenas alguns minutos. Já através de CPNs o tempo de processamento era bem maior;

- Os recursos gráficos e resultados do Promodel eram superiores e mais diretos do que em CPNs. Para obter os mesmos resultados através de CPNs a complexidade de programação era bem maior;

- CPNs possibilitam conclusões estruturais precisas sobre o processo, fundamentadas matematicamente. Já com o Promodel sabe-se que existe um gargalo, por exemplo, mas não se sabe o motivo exato do mesmo;

- Os atributos do modelo são facilmente alterados no modelo construído no Promodel, ao contrário do modelo em CPNs.

Neste trabalho, observou-se que o modelo construído utilizando simulação mostrou resultados mais eficazes do que CPNs, principalmente devido ao grau de complexidade do mesmo (quantidade de etapas e organização do tipo flow shop). O modelo de processo com quem o SAD interage para obter todas as respostas apresentadas ao gestor foi feito utilizando simulação. As funções disponíveis para representação do modelo dentro do simulador estão prontas e requerem um menor grau de complexidade de programação. Além disto existem uma série de gráficos que auxiliam na interpretação dos resultados numéricos obtidos. Já em CPNs a complexidade de representação é bem maior, visto que na literatura a maioria dos exemplos de representação de processos se restringe a pequenas células de sistemas flexíveis de manufatura e exemplos de processos industriais de pequenas dimensões. Por este motivo apenas as propriedades estruturais de CPNs foram utilizadas para complementar os resultados obtidos pelo SAD. Salienta-se que o mesmo trabalho poderia ser feito com ambas as técnicas de modelagem, mas o trabalho de programação e representação gráfica com CPNs seria bem maior. 


\section{CONCLUSÃO}

O objetivo do trabalho foi satisfeito, uma vez que foi construído um Sistema de apoio à decisão híbrido, utilizando as principais propriedades das técnicas de simulação e CPNs, contando ainda com um SE para auxílio em sua utilização. A interface criada entre o sistema e os conceitos/técnicas utilizados facilitou a utilização desta ferramenta pelos gestores, sem que estes tenham conhecimento das camadas envolvidas no protótipo. Com o levantamento das necessidades e diagnóstico feitos foi possível a construção de um modelo genérico da planta de fabricação, dando uma maior confiança e qualidade nas decisões a serem tomadas.

As técnicas de modelagem - simulação e Redes de Petri - ajudaram no direcionamento dos gestores frente às análises de desempenho, possibilitando: otimização de processos, verificação dos impactos e riscos frente às simulações, um melhor controle sobre seus objetivos de produtividade e qualidade, e por último uma melhor compreensão global da sua planta produtiva. As técnicas de modelagem utilizadas mostraram-se adequadas e corresponderam aos objetivos e expectativas estabelecidos pelos gestores. As principais características de cada técnica foram exploradas levando em consideração as aplicações típicas destas em ambientes de manufatura. A simulação mostrou-se eficiente para auxiliar na compreensão e melhorar o desempenho do sistema modelado, enquanto que as CPNs foram adequadas para análise estrutural do modelo e para complementar resultados obtidos por simulação. A fase de análise de diferentes cenários para o processo de fabricação de matrizes (caso estudado) avaliou a utilidade do SAD e validou a estrutura do protótipo. A ferramenta desenvolvida auxiliou gestores a configurar um ambiente de testes para entender relações de causa e efeito em suas decisões, melhorar o desempenho dos processos e sugerir alterações baseadas em resultados comprovados pela validação do modelo (Hennemann, 2004).

A próxima fase de desenvolvimento do sistema irá na direção de melhorar o nível de inteligência do SE, para que se possa sugerir cenários ideais para os dados fornecidos como parâmetros de entrada e obtidos como resultados, registrados no histórico, já que na versão atual ele apenas auxilia na utilização e interpretação dos resultados do SAD. Também serão propostas metodologias de coleta automática e contínua de dados em plantas industriais utilizando tecnologias do tipo RFID (identificação por rádio freqüência), para que a tarefa de coleta seja simplificada e, com isto, aprimorar a precisão dos modelos de processo. Além disto, o sistema desenvolvido será testado em outros processos produtivos reais para comprovar a sua generalidade.

\section{REFERÊNCIAS}

Anderson, M. and G. Olsson (1998). A Simulation Based Decision Support Approach for Operational Capacity Planning in a Customer Order Driven Assembly Line. Proceedings of the 1998 Winter Simulation Conference (December), vol. 2, pp. 935-941.

Askin, R.G. and C.R. Standridge (1993). Modeling and analysis of Manufacturing Systems. John Wiley \& Sons, Inc., N.Y., USA.

Banks, J., J.S. Carson and B.L. Nelson (1996). Discret-Event System Simulation. Prentice-Hall, Inc., Upper Saddle River, N.J., USA.

Bohanec, M. (2001). What is Decision Support. In Proceedings of the 4th International Multi-conference Information Society (October), vol. A, pp. 86-89.

Descotes, G.B. (1994). Coloured Petri nets: a same tool for modelling, simulation and control of manufacturing systems. IEEE International Conference on Systems, Man, and Cybernetics 'Humans, Information and Technology (October), vol. 2, pp. 1677-1682.

Dicesare, F., G. Harhalakis, J.M. Proth, M. Silva, and F.B. Vernadat (1993). Practice of Petri Nets in Manufacturing. Chapman\&Hall, London.

Groover, M.P. (2001). Automation Production Systems and Computer-Integrated Manufacturing. 2.Ed.Prentice Hall, New Jersey.

Hennemann, F.A. (2004), Uma Abordagem Híbrida para Sistemas de Apoio à Decisão utilizando Redes de Petri e Técnicas de Simulação, Dissertação de Mestrado, Depto. de Engenharia Elétrica, UFSC,FlorianópolisSC.

Jeng, M.D., S.W. Chou and C.L. Chung (1997). Performance Evaluation of an IC Fabrication System Using Petri Nets. IEEE International Conference on Systems, Man, and Cybernetics 'Computational Cybernetics and Simulation' (October), vol. 1, pp. 269-274.

Jensen, K. (1996). Coloured Petri Nets - Basic Concepts, Analysis Methods and Pratical Use, vol. 1. 2 ed.

Law, A.M. and W.D. Kelton (2000). Simulation Modeling and Analysis. 3. Ed. - MacGraw Hill, USA

Lia (2003). Expert Sinta Shell - Uma Ferramenta Visual Para Construção de Sistemas Especialistas. (http://www.lia.ufc.br/ bezerra/exsinta/exsintashell.htm Acessado em 20/09/2003). 
Malmborg, C.J.and L.M. Berrings (1992). A Decision Support System for the Design of Batch Manufacturing System. IEEE International Conference on Systems (October), vol. 2, pp. 1442-1447.

Matsuyama, A. and R.W. Atherton (1990). Experience in Simulating Wafer Fabs in the USA and Japan. IEEE/SEMI International Semiconductor Manufacturing Science Symposium (May), pp. 113-118.

Mielke, R.R. (1999). Applications for Enterprise Simulation. Proceedings of the Winter Simulation Conference (december), vol. 2, pp. 1490-1495.

Narciso, M.E. and M.A. Piera (2001). Coloured Petri Net Simulator: A Generic Tool for Production Planning. IEEE International Conference on Emerging Technologies and Factory Automation, vol. 2, pp. 139-147.

Piesik, P. and J. Weglarz (1999). Multicriteria Decision Support for Flexible Manufacturing Systems Using an Interactive Method. 7th IEEE International Conference on Emerging Technologies and Factory Automation (October), vol. 1, pp. 733-734.

Pool, M. and R. Stafford (1998). Optimization and Analysis of Performance in Simulation. Proceedings of the 1998 Winter Simulation Conference (December), vol. 2, pp. 1689-1691.

Reilly, J.J. and W.R. Lilegdon (1999). Introduction to FACTOR/AIM. Proceedings of the Winter Simulation Conference (December), vol. 1, pp. 201-207.

Shahraray, M.S. and M. Maeschke (1990). A Simulation Based Decision Support System. Proceedings of the 1990 Winter Simulation Conference (December), pp. 664668.

Zhang, L., L.M. Kristensen, C. Janczura, G. Gallasch, and J. Billington (2002). A Coloured Petri Net Based Tool for Course of Action Development and Analysis. Conferences in Research and Practice in Information Technology, pp. 125-134.

Zimmermann, A., K. Dalkowski, and G. Hommel (1996). A Case Study in Modeling and Performance Evaluation of Manufacturing Systems Using Coloured Petri Nets. In: Proc. 8th European Simulation Symposium, pp. 282-286. 